\title{
ACUTE BONE AND JOINT INFECTIONS IN CHILDREN: REVIEW OF THE CURRENT EVIDENCE
}

\author{
Ahmed Abdelaal ${ }^{1}$, Mohamed Osman ${ }^{1}$, Ashraf Marzouk ${ }^{1}$, Emmanouil Liodakis ${ }^{2}$, Mohamed \\ Kenawey $^{1\left(^{*}\right)}$

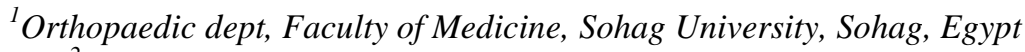 \\ ${ }^{2}$ Trauma dept., Hannover Medical School, Hannover, Germany. \\ *E-mail: mohamed.kenawey@mft.nhs.uk
}

\begin{abstract}
Acute bony and joint infections are serious musculoskeletal affections that are mainly encountered during childhood. A multi-disciplinary approach is required for prompt diagnosis and treatment to decrease long-term morbidity and loss of function. Involvement of multiple specialties including paediatricians, orthopaedic surgeons, infectious disease, emergency medicine, intensive care, laboratory and radiology specialists is necessary. Diagnosis requires high level of suspicion particularly in neonates and very young children. Knowledge of the relevant bacterial pathogens, their age prevalence and antibiotic sensitivity profile are essential for successful medical treatment. Antibiotic resistance and the emergence of virulent bacterial strains like CA-MRSA and PVL S. aureus are other challenging aspects of musculo-skeletal infections. Adequate antibiotic therapy duration is very controversial aspect in bony and joint infection and should be individualized according to the virulence of the infecting organism; the clinical improvement and laboratory follow up. Finally, surgical treatment might be needed in particular for acute joint infections and should be done timely without delay when indicated.
\end{abstract}

Keyword: Ilizarov, Varum, Valgum, Osteotomy, Correction

\section{Introduction}

Both acute bacterial osteomyelitis and pyogenic septic arthritis are serious paediatric infectious conditions. Acute bacterial osteomyelitis in children is a potentially limb and life- threatening condition that requires prompt diagnosis and treatment [1-5]. Its clinical presentation and imaging can be highly variable and culture can be negative in 34 to $60 \%$ of histologically proven osteomyelitis cases. Delayed treatment can result in overwhelming sepsis, chronic infection, bony deformity, and growth retardation $[1,6]$. Complications generally arise within one year of discharge and the rate ranges from 10 to $25 \%$. Risk factors for complications include infection with Staphylococcus aureus, disease in infancy, and a 4-to-5-day delay from symptom onset to treatment $[7,8]$. On the other hand, septic arthritis is a severe, rapidly progressive erosive joint disease. It accounts for approximately $0.25 \%$ of paediatric hospital admissions [9]. In a study of 471 children by Jackson et al, almost half of the patients were younger than 2 years of age. Another recent multicenter study by Calvo et al [10] reported a much older age presentation and was about 42 months. Single joint infection occurs in $94 \%$ of children, most commonly involving the hip, knee, ankle, elbow, wrist, and shoulder [11,12]. 


\section{Relevant Microbiology}

A very wide variety of pathogenic bacteria can cause osteomyelitis and septic arthritis. The typical pathogens vary according to the age of the patient. Staphylococcus aureus is involved in cases of all age groups, in addition to group B Streptococcus, E. Coli, Haemophilius influenza in neonates and Kingella kingae, Strep pneumonia, N. meningitides in early childhood up to 3 years and group A $\beta$-haemolytic streptococcus in childhood and adolescent
(3-18 years). An increasing problem is infection with methicillin-resistant staphylococcus aureus MRSA across all age ranges [9]. S. aureus is the main accused organism in the pathogenesis of septic arthritis as well, though the spectrum of causative bacteria includes Brucella melitensis, H. influenzae, K.kingae, N. Meningitidis, and N. Gonorrhoeae [13,14].

\section{Pathogenesis and Clinical Presentation}

There are four distinct chronological types of pediatric osteomyelitis, according to the time of onset, clinical presentation and response to treatment: acute hematogenous osteomyelitis (AHO), subacute osteomyelitis and chronic osteomyelitis,. Acute haematogenous osteomyelitis has a predilection for metaphysis of long bones especially of the lower extremity (27\% femur, $26 \%$ tibia, $9 \%$ pelvis, $8 \%$ humerus) because of the slow sinusoidal blood flow predisposing to bacterial colonization at this point [1]. Moreover, vessel-associated phagocytes are lacking in these sections. This promotes bacterially induced endothelial damage with secondary thrombosis in the area of the otherwise extremely wellperfused metaphyses [15]. A collection form in the metaphyses leading to resorption of the localized bone as a result of combination of osteoblast death and osteoclast activation with release of prostaglandin E2 which lead to further resorption [16]. Pus may penetrate through the thin metaphyseal cortex resulting in elevation of the periosteum and formation of subperiosteal abscess which deprives the cortex of its periosteal blood supply and causes necrosis and sequestration in the absence of effective treatment. The periosteum around the sequestrum still has an osteogenic activity and deposit a shell of new bone called involucrum. In neonates, there are vascular channels passing from the metaphysis into the epiphysis, therefore, oste-omyelitis in the metaphysis can result in thrombosis of these vessels leading to septic epiphysitis and subsequent osteonecrosis of the epiphysis [1]. Clinical presentations of acute osteomyelitis include general and local manifestation. Fever is the commonest symptom which is of acute onset in AHO and usually of high grade. In neonate, osteomyelitis may not be presented by fever in about $50 \%$ of cases. Anorexia, irritability, fatigue and lethargy are less common. Local manifestation includes pain, localized tenderness, swelling, warmth, inability to use limbs and redness. Traumatic determinism is commonly met with a history of previous trauma to which the symptoms might be attributed. However, trauma may predispose to infection through decreased resistance of injured tissues [17]. Synovial seeding during bacteremia is the most common mechanism of acute septic arthritis [18]. Other mechanisms include direct inoculation from penetrating injuries or spread from nearby metaphyseal osteomyelitis. This is particularly prevalent in joints where the metaphysis is partially intracapsular like the shoulder, hip, elbow (proximal radius) and ankle (distal fibula). Pathogenesis starts by cytokines release from macrophages, polymorphonuclear leukocytes and synovial cells into the joint space. Cytokines involved in this process 
include IL-1 $\beta$, IL-6, TNF- $\alpha$, immunoglobulin $\mathrm{G}$, and lysosomal enzymes. This results in early loss of proteoglycan subunits from the cartilage matrix, which might be severe as early as 2 to 5 days after the onset of infection despite the lack of visible cartilage morphologic changes. It remains unclear whether joint cartilage is able to restore normal proteoglycan content after elimination of the bacterial infection and before the onset of collagen loss or not [19]. Clinical picture of septic arthritis has constitutional and local findings. Fever, malaise, and anorexia are the main general constitutional manifestation and almost the same as acute osteomyelitis. Limping, loss of joint range of motion ROM, inability to weight bear, and pseudo-paralysis in the neonate are other presentations. In general, septic arthritis should be considered in any ill-appearing child with a clinical history of atraumatic limitation of joint mobility and has the physical findings of joint irritability [2].

\section{Laboratory Investigations}

Clinical suspicion should be combined with laboratory tests and imaging to confirm the diagnosis. Complete blood counts with differential leucocytic count, ESR, CRP and blood cultures should be ordered. Blood cultures are positive in up to $50 \%$ of cases with AHO [21]. Serial CRP measurement is an effective test to monitor response to treatment. CRP is elevated early in the disease process and levels normalise within a week of effective treatment [22]. Saavedra et al found that elevated CRP level (>4 mg/dl) was seen in $86 \%$ of children with AHO caused by MRSA (Methicillinresistant Staphylococcus aureus) and in only $58 \%$ of children with acute MSSA (Methicillin-sensitive Staphylococcus aureus) osteomyelitis [23]. In contrast, ESR may take 24-48 hours to elevate and 3 weeks to normalise [22]. The more recent advance in the diagnosis of bacterial infection is the serum procalcitonin (PCT)
Thorough history acquisition and physical examination are mandatory to narrow the wide diversity of differential diagnosis of septic arthritis which includes inflammatory and developmental conditions, transient synovitis, reactive arthritis, juvenile rheumatoid arthritis, Kawasaki's syndrome, Henoch-Schönlein purpura, rheumatic fever, slipped capital femoral epiphysis, trauma, neoplasia, Lyme arthritis, and Legg-CalvéPerthes syndrome or nearby infections as osteomyelitis, pyomyositis, septic bursitis, cellulitis, and abscesses. Clinical dilemma is often met when transient synovitis of the hip is the possible differential diagnosis. Transient synovitis is one of the most common causes of hip pain in children, responsible for up to $0.9 \%$ of pediatric emergency room visits each year and proper diagnosis can decrease unnecessary invasive procedures and hospitalization [20]. On the other hand, failure to correctly identify septic arthritis may result in poor longterm outcomes.

level. In a prospective study of 44 children by Butbul-Aviel et al [24] evaluating the diagnosis musculoskeletal infections, elevated PCT $(>0.5 \mathrm{ng} / \mathrm{mL})$ was useful in the diagnosis of osteomyelitis but not septic arthritis and was more effective than CRP, ESR or WBC. In cases of septic arthritis, Kocher's criteria (non-weight bearing, temperature $>38.5^{\circ} \mathrm{C}$, ESR $>40$ and $\mathrm{WBC}>12000$ cells $/ \mathrm{mm}^{3}$ ) were shown to have high predictive value (up to $99 \%$ ) in differentiating septic arthritis from transient synovitis [25]. However, Luhmann et al [26]. reported that the predictive probability for Kocher's criteria might be as low as $59 \%$. Joint aspiration or arthrocentesis is another essential step in the diagnosis of a septic arthritis and it could be done as a part of the physical examination and the aspirate should be sent for laboratory evaluation. A WBC count $>50,000 / \mathrm{ml}$ with $>75 \%$ polymorphonuclear 
cells is suggestive of sepsis. A gram stain may confirm the presence of organisms, however, a positive gram stain is noted in only $30 \%$ of cases of confirmed joint sepsis [9]. Synovial fluid glucose and protein levels might be obtained and compared to the serum levels. A high protein content and low glucose concentration $(<33 \%$ of serum glucose $)$ are

\section{Imaging Studies}

Plain radiographs is the primary imaging investigation for both osteomyelitis and pyogenic arthritis and soft tissue swelling may be seen early but bone changes in case of osteomyelitis often take two weeks to become evident [1]. Early in the course of septic arthritis, radiographs are usually negative, apart from slight widened joint space, fig. (1). CT studies provide specific anatomic information about the status of bony infection through localization of sequestra, the presence of intra-osseous gas and detection of subperiosteal abscess formation and aid in planning subsequent treatment [28]. Ultrasonography may not play a major role in the diagnostic algorithm of osteomyelitis, apart from detecting the existence of subperiosteal abcess and differentiating between acute osteomyelitis and septic arthritis, but it is the corner stone in the diagnosis of septic arthritis, especially in the deep-seated hip joint. Soft tissue ultrasonography can detect the presence of joint effusion, differentiate the condition from transient synovitis of the hip, as well as guiding the joint aspiration or arthrocentesis [27]. Comparative ultrasonography of both hip joints is mandatory when ultrasonographic finding are equivocal or not conclusive. Technetium -99 (TC 99) bone scan is a good investigation for localization of osteomyelitis, especially useful in neonates and in children with multifocal osteomyelitis, but it may be negative in the first 24 hours [29,30]. Increased uptake in the affected region is characteristic of septic arthritis. Different methods are available to perform joint arthrocentesis safely under ultrasound and fluoroscopic guidance. Although blind aspiration based on anatomic landmarks can be attempted, it is not recommended because it is impossible to confirm an intra-articular location in the case of a negative aspirate [27].

the norm, however, a cold scan should be managed aggressively. It frequently represents severe osteomyelitis with a higher necessity of surgical intervention and prolonged medical management [30]. MRI may be considered as the gold standard for diagnosing osteomyelitis but its use can be reserved for the more complicated cases. Bony infections appear as reduced signal on T1 sequence and high signal intensity area on T2- weighted images. Careful correlation with clinical picture is needed as fracture, bone bruise and bone infarcts can have a similar appearance. Fat suppression sequence post gadolinium contrast highlights marrow changes and abscesses and demonstrates ring enhancement [9]. MRI can be used to identify intra-osseous, subperiosteal and soft-tissue abscesses, thus enabling early abscess drainage without exposure to radiation, fig, (2). The sensitivity and specificity of MRI for the diagnosis of osteomyelitis range between $82-100 \%$ and $75-96 \%$ respectively [28]. In cases of septic arthritis, when ultrasonography fails to prove the diagnosis and in the presence of high clinical suspicion or failed aspiration, MRI can be of great value. Both T1 and T2 weighted images are helpful in determining the presence of joint effusions. Furthermore, fat suppression sequence post gadolinium contrast highlights other associated finding as marrow changes and the presence of abscesses with its typical ring enhancement [9]. 


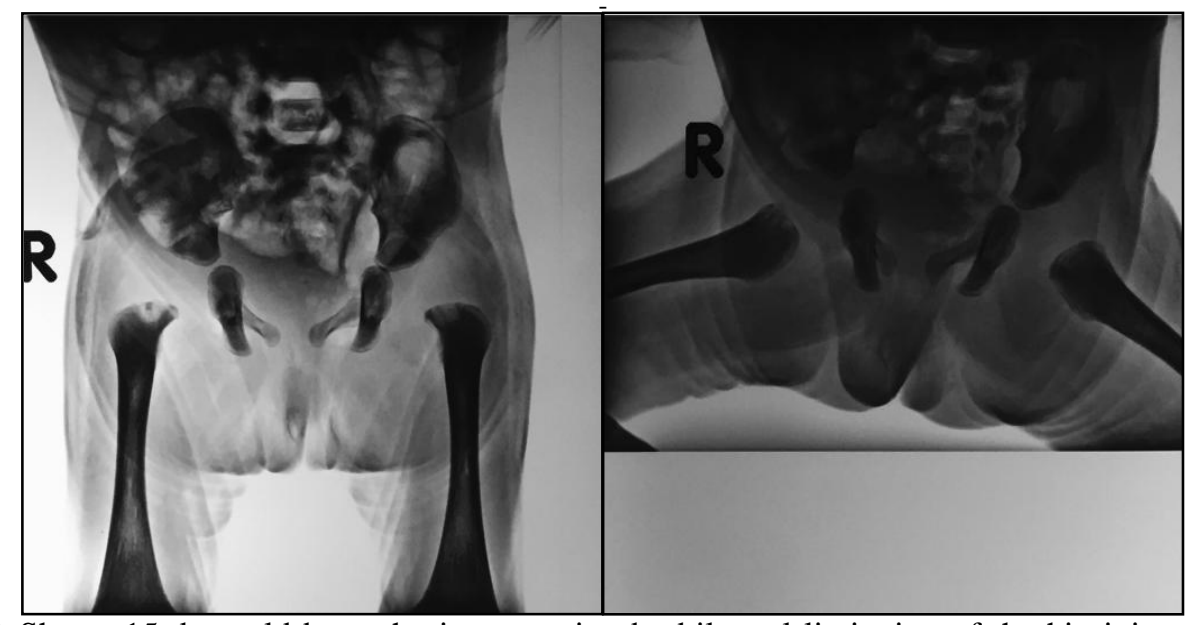

Figure (1) Shows 15-days old boy who is presenting by bilateral limitation of the hip joint motion and pseudoparalysis. His $\mathrm{x}$-ray shows bilateral proximal femoral osteomyelitis with widened hip joint spaces and bilateral pathologic hip dislocation due to secondary septic arthritis.
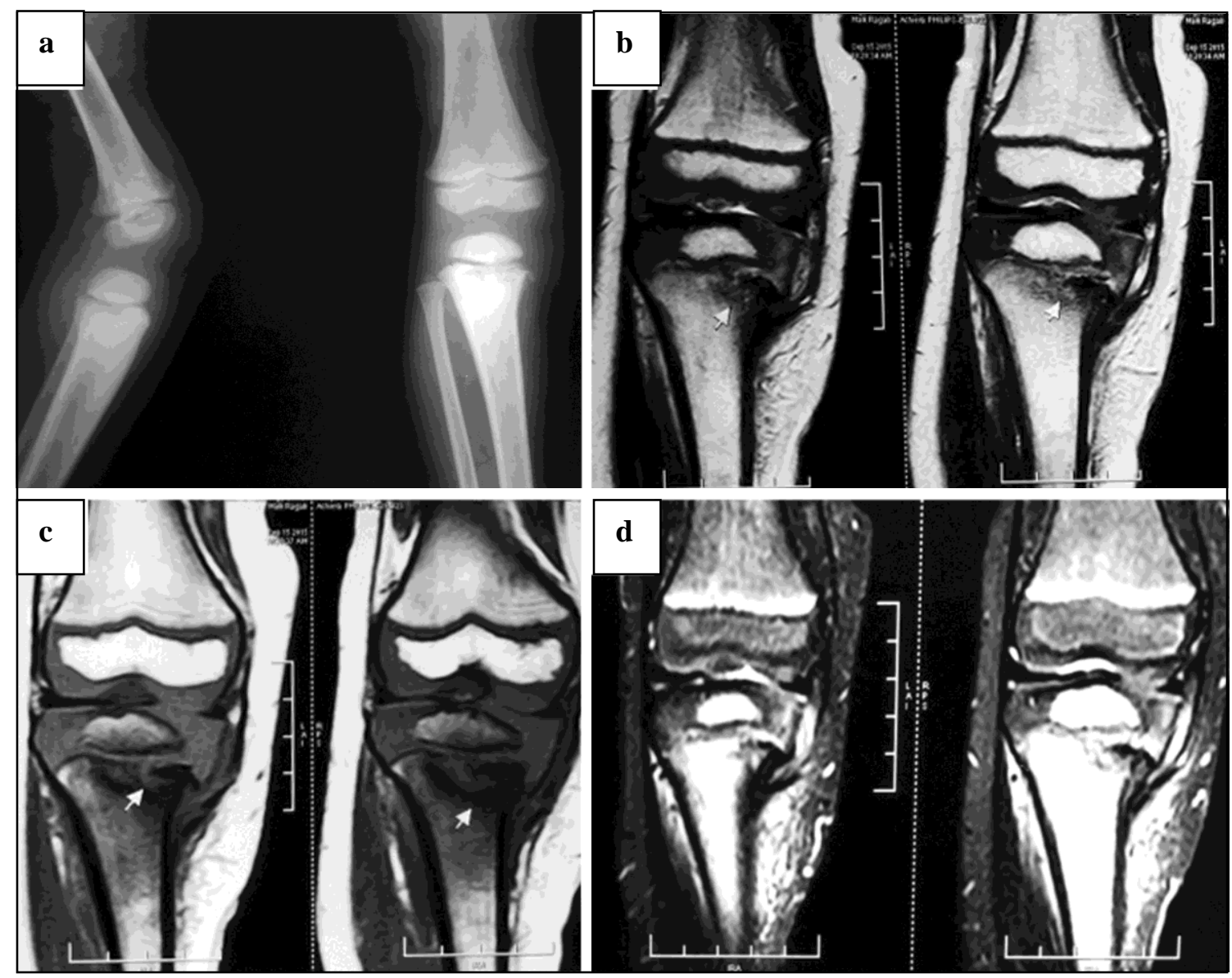

Figure (2) Shows a patient who is suffering from acute osteomyelitis of the proximal tibia; a. plain x-ray shows rarefaction in the medial proximal metaphyseal area of the tibia with faint periosteal reaction, $\underline{\mathbf{b}}$., $\underline{\mathbf{c}} . \& \underline{\mathbf{d}}$. T2, T1 and STIR MRI images respectively showing the proximal tibial metaphyseal marrow oedema and high signal intensity area together with a medial subperiosteal abscess formation.

\section{Choice of Antibiotic Therapy}

Once diagnosis of septic arthritis or acute osteomyelitis is established or has become the most likely diagnosis, parenteral empirical antibiotic should start immediately depending on prevalence patterns of certain organisms in certain community with 
consideration of emergence of drug resistance and the age of the patient as well as the immunization status. Antibiotics that have proven efficacy against $S$. aureus bone and joint infections include nafcillin, clindamycin, first-generation cephalosporins and vancomycin $[31,33]$. If MRSA is not a concern in a community with less than $10 \%$ CA-MRSA determined by local antibiograms, then empiric therapy for children older than 3 months of age should be an anti-staphylococcal penicillin (nafcillin and oxacillin) or first-generation cephalosporin (cefazolin). These agents are effective against MSSA and other causes of AHO including $\mathrm{S}$. pyogenes and $\mathrm{K}$. kingae. In communities with $10 \%$ or more CA-MRSA, vancomycin or clindamycin (if local clindamycin resistance rates are $\leq 25 \%$ ) should be used for empiric treatment of children [4]. Preferably, the antibiotic should be bactericidal with some effect against the slow-growing organisms that are protected within a biofilm (eg, coagulasenegative $\mathrm{S}$ aureus [CONS]). Rifampin fulfills these requirements; however, this agent should never be used alone because of the rapid development of bacterial resistance to the drug [34]. New antimicrobial agents have also become available for the treatment of infections caused by MRSA. These include linezolid (an oxazolidinone antibiotic) and daptomycin (a lipopeptide antibiotic). In a study included 13 children treated with linezolid as stepdown or alternative therapy for osteoarticular infections, 11 of the 13 children did well, with a mean duration of therapy of 20 days and during the course of therapy, two children developed anemia. Myelosuppression is a known side effect of prolonged therapy with linezolid, as are optical and peripheral neuropathies. For this reason, a treatment course of longer than 21 days is not recommended [4,35]. Other treatment algorithm particularly in cases with acute septic arthritis depend on the age of the patient [9]. In the first 8 weeks of life, nafcillin, oxacillin plus gentamicin, or a third generation cephalosporin i.e., cefotaxime or ceftriaxone is commonly used for Staph aureus, Streptococci, Enterobacteriaceae, Klebsiella or Candida species. Later, after starting the immunization schedule of the infant, first generation cephalosporinns might be used in immunized child, e.g, cefazoline or nafcillin or oxacillin. In non-immunized child, nafcillin or oxacillin plus a second or third generation cephalosporin, (cefuroxime or cefotaxime respectively) can be used as the common pathogens till $3^{\text {rd }}$ year of life are $S$. aureus, Kingella kingae, Streptococcus pneumoniae, Neisseria meningitides or Haemophilus infuenzae type $b$. In older children up to twelve years, Staph aureus and group A beta hemolytic streptococci, are the commonest pathogens and treated using the same protocols. In adolescent, $S$. aureus, group A beta hemolytic streptococci and Neisseria gonorrhoeae are the most prevalent organisms and treatment options expands to include doxycycline to treat disseminated gonococcal infection $[9$,$] .$

\section{Duration of Systemic Antibiotic Administration}

Duration of parenteral administration of antibiotics is a controversial issue. Traditionally, up to six weeks of parenteral antibiotic treatment was the rule, however recent studies revealed comparable results with a shorter course and early switch to oral treatment. Once acute phase reactant had subsided and shooting ESR and CRP are decreasing, shift to oral treatment could be safely carried out. This would reduce hospital stay, cost, morbidity, and inconvenience to families [36]. CRP typically returns to baseline within a week of effective therapy and many authors advocated its use as a guide to the switch from parenteral to oral antibiotic administration [31]. Chou et al reported that a 50\% reduction in CRP levels was associated with clinical improvement in patients with acute bacterial osteoarticular infections 
and can be used for the transition to oral antimicrobial therapy [37]. Arnold et al [38] showed $99.5 \%$ success in 194 pediatric patients with acute bacterial osteomyelitis who were switched to oral therapy based on the improvement of the clinical picture and CRP $<2 \mathrm{mg} / \mathrm{dL}$ and only one patient experienced treatment failure due to retained infected bone fragment in the hip joint. Kocher et al [39] reported an early switch to oral antibiotics after 72 hours of intravenous administration, as long as there was a good response to the initial treatment. In general, the duration of antibiotic therapy for most cases of uncomplicated septic arthritis is approximately 4 weeks, although shorter treatment courses have been described. In a systematic review of 12 prospective studies comparing the outcome of a short ( $<7$ days) versus a long ( $>7$ days) course of intravenous antibiotic treatment for $\mathrm{AHO}$ caused primarily by
MSSA in children 3 months to 16 years of age, an overall cure rate was $95.2 \%$ for the short course compared to $98.8 \%$ for the longer course at 6 months [40]. Peltola et al conducted a prospective randomized study in pediatric patients with acute osteomyelitis caused primarily by MSSA strains and found that oral antibiotics (clindamycin or a first-generation cephalosporin) for 20 days had the same efficacy as 30-days treatment after an initial intravenous treatment of two to four days in both groups [41]. Similar results were obtained by Jagodzinski et al in 70 children between two weeks and 14 years of age suffering from acute osteomyelitis or septic arthritis who were treated for three to five days with high dose intravenous therapy and then with oral therapy for three weeks [42]. In case with MRSA or PVL S. aureus infections, four to six weeks of treatment are recommended [43].

\section{Dexamethasone in Acute Septic Arthritis}

In 1996, Sakiniene et al [44] reported the use of corticosteroids with antibiotic treatment to decrease the severity of septic arthritis and lower the mortality rate in mice with experimental S. aureus arthritis. Many studies had investigated this hypothesis and reported a chondroprotective effect of the drug when administered early and in conjunction with systemic

\section{Surgical Treatment}

In patients with acute hematogenous osteomyelitis, conservative treatment with antibiotic administration should be the mainstay treatment as long as there is clinical and laboratory improvement. Evidence of pus formation or sequestration indicates the necessity of surgical drainage [31]. In 1983, S. Nade proposed his five principles for the treatment of acute osteoarticular infections that are still applicable today: (1) an appropriate antibiotic is effective before abscess formation; (2) antibiotics do not sterilize avascular tissues or abscesses and such areas require surgical removal; (3) if such removal is antibiotics in animal models [45,46]. In a double blind randomized controlled trial of 123 children with hematogenous septic arthritis, Odio et al [47] showed that 4days dexamethasone therapy shortened the duration of acute symptoms and reduced the residual dysfunction at the end of therapy and during follow up compared to placebo (saline).

effective, antibiotics should prevent their re-formation and primary wound closure should be safe; (4) surgery should not damage further already ischemic bone and soft tissue; and (5) antibiotics should be continued after surgery [48]. Surgery also makes it possible to obtain biological samples that are useful for identifying the etiologic agent and guiding the selection of the correct antibiotic. On the other hand, surgical intervention should be performed as a part of the initial treatment for septic arthritis, along with intravenous administration of an appropriate antibiotic. When to interfere is a debatable issue, though many 
surgeons accept that a high cellular content on joint fluid aspiration $>50.000 / \mathrm{CC}$, or presence of bacteria on gram stain examination of the aspirate are indications for surgical intervention [49]. Other surgeons would prefer to repeatedly aspirate the joint along with the administration of parenteral antibiotics. However, early aggressive surgical intervention is the widely accepted approach to decrease the formation of fibrinous loculations, synovitis and creeping pannus [50]. Different surgical modalities are available to decompress the affected joints with septic arthritis ranging from

\section{References}

[1] Dartnell, J., Ramachandran, M., Katchburian, M. (2012). Haematogenous acute and subacute paediatric osteomyelitis. J. Bone Joint Surg Br. 94: 584-595.

[2] Dodwell, E. (2013). Osteomyelitis and septic arthritis in children: Current concepts. Current Opinion in Pediatrics. 25: 58-63.

[3] Faust, S., Clark, J., Pallett, A., et al. (2012). Managing bone and joint infection in children. Archives of Disease in Childhood. 97(6):545-53.

[4] Howard-Jones, A., Isaacs, D. (2013). Systematic review of duration and choice of systemic antibiotic therapy for acute haematogenous bacterial osteomyelitis in children. J. of Paediatrics and Child Health. 49: 760-768.

[5] Peltola, H., Unkila-Kallio, L., Kallio, M. (1997). Simplified treatment of acute staphylococcal osteomyelitis of childhood. Pediatrics. 99: 846-850.

[6] Bonhoeffer, J., Haeberle, B., Schaad, U., et al. (2001). Diagnosis of acute haematogenous osteomyelitis and septic arthritis: 20 years experience at the University Children's Hospital Basel. Swiss Medical Weekly. 131: 575-581.

[7] Roine, I., Arguedas, A., Faingezicht, I., et al. (1997). Early detection of sequelaprone osteomyelitis in children with use of simple clinical and laboratory criteria. aspiration in emergency room to open arthrotomy or arthroscopic drainage and lavage or synovectomy [49]. Arthroscopic drainage and lavage with or without synovectomy gradually replaces the conventional open arthrotomy or open synovectomy. Arthroscopic assistance would allow better intraarticular visualization, enables the surgeon to remove the fibrinous aggregates and to debride aggressive synovitis. One important advantage for arthroscopic procedures are being minimally invasive with faster postoperative rehabilitation. ${ }^{50}$

Clinical Infectious Diseases. 24: 849853.

[8] Wu, J., Gorbachova, T., Morrison, W., et al. (2007). Imaging-guided bone biopsy for osteomyelitis: Are there factors associated with positive or negative cultures? American J. of Roentgenology. 188: 1529-1534.

[9] Copley, L., Herring, J. (2013). Infections of the Musculoskeletal System. In: Herring, J. (ed.) Tachdjian's Pediatric Orthopaedics: From the Texas Scottish Rite Hospital for Children, $5^{\text {th }}$ ed. Philadephia, Elsevier Health Sciences, pp. 1024-1078.

[10] Calvo, C., Núñez, E., Camacho, M., et al. (2016). Epidemiology and Management of Acute, Uncomplicated Septic Arthritis and Osteomyelitis: Spanish Multicenter Study. The Pediatric Infectious Disease J. 35: 1288-1293.

[11] Control CfD, (2004). Prevention: Osteomyelitis/septic arthritis caused by Kingella kingae among day care attendees--Minnesota 2003. MMWR Morbidity and Mortality Weekly Report. 53:241.

[12] Osman, A., Govender, S. (1995). Septic sacroiliitis. Clinical Orthopaedics and Related Research. 313: 214-219.

[13] Howard, A., Viskontas, D., Sabbagh, C. (1999). Reduction in osteomyelitis and septic arthritis related to Haemophilus 
influenzae type B vaccination. $\boldsymbol{J}$. of Pediatric Orthopaedics. 19 (6): 705709.

[14] Vinod, M., Matussek, J., Curtis, N., et al. (2002). Duration of antibiotics in children with osteomyelitis and septic arthritis. J. of Paediatrics and Child Health. 38: 363-367.

[15] Mousa, H-L. (1997). Evaluation of sinus-track cultures in chronic bone infection. Bone \& Joint Journal; 79: 567-569.

[16] Speers, D., Nade, S. (1985). Ultrastructural studies of adherence of Staphylococcus aureus in experimental acute hematogenous osteomyelitis. Infection and Immunity. 49: 443446.

[17] Morrissy, R., Haynes, D. (1989). Acute hematogenous osteomyelitis: A model with trauma as an etiology. J. of Pediatric Orthopaedics. 9: 447456.

[18] Saslaw, M., Mishra, S., Green, M., et al. (1999). Suppurative arthritis complicating otitis media. The Pediatric Infectious Disease Journal. 18: 475-476.

[19] Riegels-Nielsen, P., (1987). FrimodtMöller, N., Jensen, J. Rabbit model of septic arthritis. Acta Orthopaedica Scandinavica. 58: 14-19.

[20] Do TT. (2000). Transient synovitis as a cause of painful limps in children. Current Opinion in Pediatrics. 12: 4851.

[21] Harik, N., Smeltzer, M. (2010) Management of acute hematogenous osteomyelitis in children. Expert Rev Anti Infect Ther. 8: 175-181.

[22] Stans, A. (2014). Musculoskeletal Infection. In: Weinstein, S., Flynn, J. (eds.) Lovell and Winter's Pediatric Orthopaedics, $7^{\text {th }}$ ed. Lippincott Williams \& Wilkins, Vol. 1, pp. 369-425.

[23] Saavedra-Lozano, J., Mejías, A., Ahmad N, et al. (2008). Changing trends in acute osteomyelitis in children: Impact of methicillin-resistant Staphylococcus aureus infections. $\boldsymbol{J}$. of Pediatric Orthopaedics. 28: 569575.

[24] Butbul-Aviel, Y., Koren, A., Halevy, R., et al. (2005). Procalcitonin as a diagnostic aid in osteomyelitis and septic arthritis. Pediatric Emergency Care. 21: 828-832.

[25] Kocher, M., Zurakowski, D., Kasser, J. (1999). Differentiating between septic arthritis and transient synovitis of the hip in children: An evidencebased clinical prediction algorithm. J. Bone Joint Surg Am. 81: 16621670.

[26] Luhmann, S., Jones, A., Schootman, M., et al. (2004). Differentiation between septic arthritis and transient synovitis of the hip in children with clinical prediction algorithms. J. Bone Joint Surg Am. 86-A: 956-962.

[27] Dörr, U., Zieger, M., Hauke, H. (1988). Ultrasonography of the painful hip. Pediatric Radiology. 19: 36-40.

[28] Pineda, C., Vargas, A., Rodríguez, A. (2006). Imaging of osteomyelitis: Current concepts. Infectious Disease Clinics. 20: 789-825.

[29] Carr, A., Cole, W., Roberton, D., et al. (1993). Chronic multifocal osteomyelitis. Bone \& Joint Journal; 75: 582-591.

[30] Pennington, W., Mott, M., Thometz, J., et al. (1999). Photopenic bone scan osteomyelitis: A clinical perspective. J. of Pediatric Orthopaedics; 19: 695698.

[31] Feigin, R., Demmler-Harrison, G., Kaplan, S. (2009). Textbook of Pediatric Infectious Diseases, Elsevier.

[32] Gutierrez, K. (2005). Bone and joint infections in children. Pediatric Clinics of North America. 52: 779-794.

[33] Lew, D., Waldvogel, F. (2004). Osteomyelitis. The Lancet. 364: 369-379.

[34] Kaandorp, C., Dinant, H., van de Laar, M., et al. (1997). Incidence and sources of native and prosthetic joint infection: A community based prospective survey. Annals of the Rheumatic Diseases. 56: 470-475. 
[35] Chen, C-J., Chiu. C-H., Lin, T-Y., et al. (2007). Experience with linezolid therapy in children with osteoarticular infections. Pediatr Infect Dis Journal 26: 985-988.

[36] Kim, H., Alman, B., Cole, W. (2000). A shortened course of parenteral antibiotic therapy in the management of acute septic arthritis of the hip. J. of Pediatric Orthopaedics. 20: 44-47.

[37] Chou, A., Mahadev, A. (2016). The use of C-reactive protein as a guide for transitioning to oral antibiotics in pediatric osteoarticular infections. $\boldsymbol{J}$. of Pediatric Orthopaedics. 36: 173177.

[38] Arnold, J., Cannavino, C., Ross, M., et al. (2012). Acute bacterial osteoarticular infections: Eight-year analysis of C-reactive protein for oral stepdown therapy. Pediatrics. 130 (4) e821e828

[39] Kocher, M., Mandiga, R., Murphy, J., et al. (2003). A clinical practice guideline for treatment of septic arthritis in children. J. Bone Joint Surg Am. 85: 994-999.

[40] Le Saux, N., Howard, A., Barrowman N., et al. (2002). Shorter courses of parenteral antibiotic therapy do not appear to influence response rates for children with acute hematogenous osteomyelitis: A systematic review. BMC Infectious Diseases. 2; 16: doi: 10.1186/1471-2334-2-16.

[41] Peltola, H., Pääkkönen, M., Kallio, P. et al. (2010). Short-versus longterm antimicrobial treatment for acute hematogenous osteomyelitis of childhood: Prospective, randomized trial on 131 culture-positive cases. The Pediatric Infectious Disease. 29: 11231128.

[42] Jagodzinski, N., Kanwar, R., Graham, K., (2009). Prospective evaluation of a shortened regimen of treatment for acute osteomyelitis and septic arthritis in children. J. of Pediatric Orthopaedics. 29: 518-525.
[43] Liu, C., Bayer, A., Cosgrove, S., et al. (2011). Clinical practice guidelines by the Infectious Diseases Society of America for the treatment of methicillin-resistant Staphylococcus aureus infections in adults and children. Clinical Infectious Diseases. 52: e18e55.

[44] Sakiniene, E., Bremell, T., Tarkowski, A., (1996). Addition of corticosteroids to antibiotic treatment ameliorates the course of experimental Staphylococcus aureus arthritis. Arthritis \& Rheumatism. 39: 1596-1605.

[45] Stricker, S., Lozman, P., Makowski, AL., et al. (1996). Chondroprotective effect of betamethasone in lapine pyogenic arthritis. J. of Pediatric Orthopaedics. 16: 231-236.

[46] Jafari, H., Sáez-Llorens, X., Paris, M., et al. (1993). Dexamethasone attenuation of cytokine-mediated articular cartilage degradation in experimental lapine Haemophilus arthritis. J. of Infectious Diseases. 168: 1186-1193.

[47] Odio, C., Ramirez, T., Arias, G., et al. (2003). Double blind, randomized, placebo-controlled study of dexamethasone therapy for hematogenous septic arthritis in children. The Pediatric Infectious Disease J. 22: 883-889.

[48] Nade, S. (1983). Acute septic arthritis in infancy and childhood. J. Bone Joint Surg Br. 65: 234-241.

[49] Bennett, O., Namnyak, S. (1992). Acute septic arthritis of the hip joint in infancy and childhood. Clinical Orthopaedics and Related Research. 281: 123-132.

[50] Stanitski, C., Harvell, J., Fu, F. (1989). Arthroscopy in acute septic knees: Management in pediatric patients. Clinical Orthopaedics and Related Research. 241:209-212. 